diabetes. $^{3}$ 5. That the glossy skin of Sir James Paget, which has been shown to depend on injury or disease of the pheripheral nerves, is sometimes seen in diabetes, and that there can be no doubt that this condition is of a somewhat similar pathological character to perforating ulcer. These considerations seem to show that it is possible-nay, more, even probable-that diabetes may sometimes play an active part in the causation of perforating ulcer.

Lastly, with regard to the operative treatment of this affection. It appears to me that the case which I have just related strongly supports Messrs. Savory and Butlin's opinion that the extreme measure (i.e., amputation of a portion or of the whole of the foot) is at best but a doubtful one; for it is evident from the diseased condition of the posterior and anterior tibial nerves that such a procedure would have led to no permanently good result in this case. If, however, amputation is decided upon, I believe it would be advisable not to adhere to hard-and-fast rules with regard to the site of the operation as laid down by various surgeons, but rather to amputate through perfectly healthy tissuesnamely, tissues external to or beyond the zone of anæsthesia so commonly present in perforating ulcer of the foot.

Nottingham.

\section{A NOTE ON THE}

TREATMENT OF PURULENT OPHTHALMIA. BY CHARLES HIGGENS, F.R.C.S.E.

SEVERE purulent ophthalmia is perhaps the most rapidly destructive disease to which the eye is subject; consequently its treatment has received a large amount of attention. It has always appeared to me that the disease could be rapidly cured if the discharge could be got rid of, or its character altered; it always seemed that it was the discharge which kept the disease going. Constantly washing away the discharge with some astringent and antiseptic lotion gave fairly good results; but the treatment was troublesome, and unless one saw to it oneself was rarely properly'carried out. Some years ago Mr. Bader first suggested that persalts of mercury killed or destroyed the pus secreted by a mucous surface; he used to employ an ointment of red oxide of mercury, atropine, and vaseline applied to the conjunctiva, and with good results. The one objection to this treatment was the severe pain it caused, which seemed to me to quite counterbalance any advantage it might have over the washing away treatment. Since cocaine has been introduced, application can be made to the eye of substances which before its introduction caused so much pain that few patients could be found who would continue to use them. I have now treated some ten or twelve cases of severe purulent ophthalmia-some of distinctly gonorrhoeal origin-by the following method, and the case reported from notes by my dresser (Mr. Andrews) is a fair specimen. 1. Wash away all discharge, and thoroughly cleanse the eyes with a 5 per cent. solution of boracic acid. 2. Apply thoroughly to the whole conjunctival surface, and fill the conjunctival sac with, an ointment composed of yellow oxide of mercury ( $16 \mathrm{gr}$.), boracic acid (20 gr.), hydrochlorate of cocaine (from $5 \mathrm{gr}$. to $10 \mathrm{gr}$.), and vaseline ( $1 \mathrm{oz}$.); and in some cases $I$ also add $2 \mathrm{gr}$. of sulphate of atropine. The ointment may be applied with a camel-hair brush, a quill, a syringe with a good wide canal, or in any way that seems most suitable, so long as care is taken that no part of the conjunctival surface escapes it. 3. Cover the eyes with lint plentifully smeared with the ointment, and bandage them. Some pain is caused by the first application, which can be prevented by using a 2 per cent. solution of cocaine before making it; after the first application the effect of the cocaine in the ointment keeps the eye numb from one application to the next, unless the applications are made at very long intervals. The eyes should be examined in about two hours. If there is any discharge the whole process must be repeated; if there is none, the bandage may be reapplied and left for another two hours. As a rule, the ointment requires to be applied every four

3 Laffon (Bordeaux), The LaxCET' (Annotation), Sept. 13th, 1886. 4 Bryant's Surgery, 4th edition. Erichsen's Surgery, published 1884. Jacobson in Heath's Dictionary of Surgery. hours for the first two days, less frequently afterwards; $b u t^{2}$ the discharge must never be allowed to collect.

Harry $W-$, aged eighteen, was admitted into the hospital on March 22nd, 1887. His history was that seven days before, March 15th, the left eye suddenly began to itch and in the course of two days the eyelids became swollen. and discharge commenced. Four days later the right eye became similarly affected; the discharge from both was very free. On admission pus was flowing freely from both eyes, there was much swelling of the eyelids, and intense congestion and swelling of the conjunctiva. There was. some difficulty in examining the corneæ; but the left was. found to be perforated, and there was an ulcer on the right. The eyes were thoroughly cleansed, and the ointment, the prescription for which is given above, was applied (the application to be repeated every four hours), and the eyes were bandaged; quinine was ordered internally and the patient put to bed. The discharge was much diminished after the first application of the ointment. On March 27thr. five days after commencing the treatment, there was very great improvement; the swelling of the eyelids had greatly subsided, the conjunctiva had flattened down and was much less congested, and the discharge, which had become less and less, had almost ceased. On April 1st there had been no discharge from the right eye, and very little from. the left. The bandage was removed from the right eye. On April 9th, all swelling and discharge had entirely gone. There was slight corneal opacity below the pupil of the. right eye. In the left eye there was an opaque flattened cicatrix - to which the iris was adherent-occupying thecentre of the cornea; the eye had good perception of light.

Remarks. - The result of treatment in this case was most marked, the only cause for regret is that the patient dic not apply sooner; had he done so, in all probability the. damage to the left eye would have been prevented. He was not suffering from gonorrhœa, but the case looked like one of gonorrhœal infection.

Brook-street, $W$.

\section{ON A CASE OF SUNSTROKE.} By A. CHEVALLIER PRESTON, M.R.C.S.

JоHN V—aged fifteen years, was brought to my house on Jan. 7th, 1887, by his mother. He had been cutting grass with a horse reaper, sitting on the machine driving, for the past two days, with no head shade but a cap, in the burning. sun. He was gradually seized with pain in the chest and back, accompanied by convulsive movements of the arms and legs and contortions of the face. Has had no previous illness.

On examining him, I found him intelligent and able to tell me what I wanted to know, but his speech was muffed. His face was congested, and there was general redness of the skin of the whole body, with beads of perspiration on. the forehead. His arms and legs were in a constant state of rhythmical convulsions. He could not protrude his tongue when asked to do so. Asked for drink. Pupils equal and slightly dilated. No unconsciousness. Pulse 100 ; temperature in axilla $99.4^{\circ}$; bowels constipated; passed urine freely. Unable to walk without assistance. Thinkıng the above symptoms were owing to the exposure to the sun in the intensely hot weather then prevalent, 1 ordered him to. be kept in a darkened room, his head to be shaved, and an ice-bag applied to the head and back of neck, with a diet of milk and slops. I also prescribed a mixture containing ten grains of bromide of potassium and five grains of hydrate of chloral, to be taken every, three hours, with five grains of calomel at bedtime.

Jan. 8th.-11 A.M.: On visiting him I found him still convulsed, with slight intermissions. No sleep had been obtaintd. The bowels had not acted. Unable to open jaw. No pain, but feels ill: Pulse 120, hard. Temperature in axilla 101'. Taking nourishment. Ordered ice-bag to be continued to the bead, milk and ice by the mouth, and gave five grains. of calomel again in powder; injected five grains of quinine hypodermically; the bromide and chloral to be continued.

9th.-3 P.M.: Has had half an hour's sleep, and says he is' in no pain. Has taken two pints of iced milk in the twentyfour hours. Had a loose motion last evening, and passed abundance of high-coloured urine. Pulse 110, still hard; 